\title{
Formulation and development of moxifloxacin nanoemulsion for ophthalmic delivery
}

*Shivani Tiwari, Neelima Goswami**, Durga Pandey*, Praveen Tahilani*, Abhishek Sharma*

*Sagar Institute of Research and Technology- Pharmacy, Bhopal, MP

** Sagar Institute of Research, Technology \& Science- Pharmacy, Bhopal, MP

Received: 10-01-2022 / Revised Accepted: 27-01-2022 / Published: 01-02-2022

\begin{abstract}
Nano emulsions formulated for the ocular drug delivery have a good scope in pharmaceutical products due to the transparency at enlarged droplet volume fraction, advanced rate of bioavailability and improved shelf life. In this study we aim to prepare \& evaluate moxifloxacin nano emulsion of for ocular delivery that will result with improved drug retention in cornea, sustained release will be achieved and dosing frequency will be reduced as compared with conventional products.

Drug identification was done by UV FTIR, melting point and solubility. Nano emulsion were prepared by standard procedure with minor modifications. Particle size was found around 380 $\mathrm{nm}$ by Malvern analyser. SEM showed droplets of spherical shape and size $300 \mathrm{~nm}$. \% entrapment was $81 \%$. Microscopy also showed drops oval to spherical in shape. Marketed formulation released $100 \%$ within 30 min where as optimized nano emulsion was released in sustained manner for more than $6 \mathrm{hrs}$. Thus it can be concluded that designed formulation can be able to improve drug activity.
\end{abstract}

\section{Keyword: Nanoemulsion, Moxifloxacin, Ocular, sustained}

\section{INTRODUCTION}

The Nanoemulsion of moxifloxacin hydrochloride is designed for ocular drug delivery for improvement of drug retention. Bacterial ocular infection is very common in eyes. Conventional aqueous solutions are very poorly absorbed due to outflow from eyes. ${ }^{[1]}{ }^{[2]}$. Nanoemulsions are novel drug delivery systems consist of emulsified oil and water systems with mean droplet diameters ranging from 50 to $1000 \mathrm{~nm}$. Usually, the average droplet size is between 100 and $500 \mathrm{~nm}$. Ocular administration of drug is primarily associated with the need to treat ophthalmic diseases. ${ }^{[3]}$ Eye is the most easily accessible site for topical administration of a medication in ocular disease. Ideal ophthalmic drug delivery must be able to sustain the drug release and to remain in the vicinity of front of the eye for prolong period of time. However many dosage form already designed for ophthalmic delivery like gel, ointment, suspension, ocuserts but all have their own limitations. ${ }^{[4]}$ Nanoemulsion is superior from these because it is less viscous than ointment, gels and

Address for Correspondence: Ms. Shivani Tiwari, Sagar Institute of Research and Technology- Pharmacy, Bhopal, MP; E-mail: tahilanipraveen@gmail.com

How to Cite this Article: Shivani Tiwari, Neelima Goswami, Durga Pandey, Praveen Tahilani, Abhishek Sharma. Formulation and development of moxifloxacin nanoemulsion for ophthalmic delivery. World J Pharm Sci 2022; 10(02): 178-182; https://doi.org/10.54037/WJPS.2022.100203 
more viscous than simple conventional solution. Moxifloxacin is a fluoroquinolone antimicrobial that fights bacteria in the body. Moxifloxacin is used to treat different types of bacterial infections of the eyes, skin, sinuses, lungs, or stomach. ${ }^{[5]}$

Tween 80 \& Span 80 is compatible excipient for ocular nanoemulsion. Tween 80 (polysorbate 80, polyoxyethylene sorbitan monooleate) is a nonionic surfactant that is widely used as an emulsifier in cosmetics, pharmaceuticals and food products. Span 80 is a non-ionic surfactant that can be used to form oil-in-water emulsions.

\section{MATERIALS AND METHODS}

Moxifloxacin received as a gift sample from Aristo Pharma Bhopal. Tween 80, Span 80, and Ethyl Oleate received from department. All chemicals used in this study were of pharmaceutical grade.

\section{Drug Solubility}

Solubility of drug was determined at various oils by equilibrium solubility method out of which solubility was found good in ethyloleate about 29 $\mathrm{mg} / \mathrm{ml}$. This is the reason to select ethyl oleate for formulation of Moxifloxacin nanoemulsion. ${ }^{[6]}$

\section{Melting point}

Melting point of drug was determined by digital melting point apparatus.

\section{pH of the Nanoemulsion}

The $\mathrm{pH}$ of emulsion was recorded at given time intervals using a digital $\mathrm{pH}$ meter ${ }^{[7]}$

\section{Determination of $\lambda$ max}

Moxifloxacin hydrochloride shows the absorbance maximum at $289 \mathrm{~nm}$ in phosphate buffer $\mathrm{pH}$ 7.4.

Which is confirmation with reported range of moxifloxacin

\section{Formulation of Nanoemulsion}

Moxifloxacin nanoemulsions $(\mathrm{O} / \mathrm{W})$ was prepared by standard method with minor modification. Various batches were prepared by changing composition of oil, water, emulsifier and coemulsifier. Appropriate quantity of deionized water, Ethyl oleate, Tween 80 and Span 80 taken for formulation. Moxifloxacin $(0.5 \%$, w/v) was dissolved in Ethyl oleate and Span 80 added in this mixture. Aqueous phase containing Tween 80 added in the nonaqueous phase. Further, it was thoroughly stirred and vortexes to obtain nanoemulsion. Sonication was done to achieve uniform small droplets size of nanoemulsion. ${ }^{[8]}$

\section{Optimization of process variables}

Nanoemulsion preparation can be affected by number of factors such as mixing time, mixing speed, sonication time and temperature used during formulation. These parameters evaluated by subjecting formulation in theses various conditions. The effects of variables were observed on the final particle size, drug loading and zeta potential during the preparation of a particular system. ${ }^{[9]}$

\section{Characterization of Nanoemulsion}

\section{Particle size distribution}

Droplets size and size distribution of emulsion system were determined using Malvern Mastersizer 2000 laser diffraction particle analyzer (Malvern instruments).

\section{Microscopy of optimized formulations}

Formulation was measured microscopically from an optical microscope (Cippon, Japan) used to observe the shape of the prepared formulation droplets.

\section{Scanning Electron Microscopy}

This analysis was performed at Chemistry department, IISER BHOPAL, ZEISS analytical SEM model 2015010 Ultra/plus using software ZEISS

\section{Drug Entrapment Efficiency}

The moxifloxacin hydrochloride loaded emulsions was centrifuged at $4^{0} \mathrm{C}$ for $35 \mathrm{~min}$ at ultracentrifuge in order to separate incorporated part of drug from the nonincorporate drug. After centrifugation the supernatant was collected and was analyzed by UV visible spectrophotometer at $289 \mathrm{~nm}$ for the unincorporated drug concentration to determine the encapsulation efficiency from total amount of drug. [10]

Entrapment efficiency $=$ Total Drug - Free Drug $\times 100$

Total Drug

\section{Viscosity determination}

The viscosity of the emulsions was analyzed using Brookfield viscometer.

\section{In-vitro drug release}

The in-vitro release profile of Nanoemulsion was performed using dialysis membrane previously soaked for 24 hours in buffer and stretched around and the one end of the tube. About one $\mathrm{ml}$ nanoemulsion was taken in pretreated membrane which were immersed into $100 \mathrm{ml}$ of phosphate buffer solution, $\mathrm{pH} 7.4$ at $37^{\circ} \mathrm{C} \&$ magnetically stirred at $50 \mathrm{rpm}$. At selected time intervals, 0, 30, $60,90,120,180,240,300,360$ aliquots were withdrawn from the release medium \& replaced with same amount of the phosphate buffer to maintain sink condition. The sample was analyzed in triplicate using UV spectrophotometer at 289 nm. ${ }^{[10]}$ 


\section{Stability Studies}

Formulations were stored at low temperature $4{ }^{\circ} \mathrm{C}$ and room temperature $25^{\circ} \mathrm{C}$ for 2 month. The creaming and the phase separation were assessed visually at given sufficient time intervals. Viscosity and $\mathrm{pH}$ change was used as a parameter to assess the effect of storage temperature in the stability of optimized formulation. ${ }^{[8]}$

\section{RESULT AND DISCUSSION}

Drug identification was done by UV given $\lambda \max$ at $289 \mathrm{~nm}$. Melting point was $240^{\circ} \mathrm{C}$ slolubility of drug was good in ethyl oleate. FTIR spectra confirmed that drug is Moxifloxacin shown in figure 1

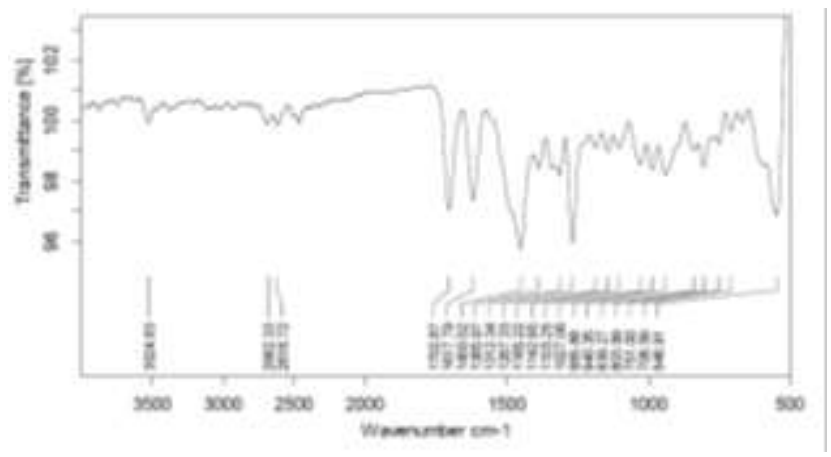

Fig. 1: FTIR spectra of Ciprofloxacin

Composition of optimized formulation (\%w/w)

Optimized formulation prepared by ethyl oleate tween 80 and span 60. Moxifloxacin $0.5 \%$ taken in all formulation as available in market in this concentration. Various batches prepared out of which based on stability entrapment and droplets size one is finalized shown in table 1

Table: 1 Composition of optimized formulation

\begin{tabular}{|l|l|}
\hline Ingredients & $\% \mathrm{~W} / \mathrm{W}$ \\
\hline Ethyl oleate & $10.00 \mathrm{ml}$ \\
\hline Span 80 & $8 \mathrm{ml}$ \\
\hline Tween 80 & $4 \mathrm{ml}$ \\
\hline Moxifloxacin $(\% \mathrm{w} / \mathrm{v})$ & 0.5 \\
\hline Water & Up to100 \\
\hline
\end{tabular}

\section{Evaluation of Nanoemulsion}

Various parameters like Homogenizer speed, sonication time and temperature were used to optimize particle size of formulation. At speed $16000 \mathrm{rpm}$ is suitable for desired particle size in nanometer rang, sonication time is 4-5 min suitable for nanometer size range. $50^{\circ} \mathrm{C}$ was found suitable for good entrapment. On increasing the sonication time, decrease in mean diameter was Observed upto $5 \mathrm{~min}$ after that there is no decreasing effect observed. So 5 min was found optimized sonication time for formulation. Temperature of the formulation system plays a very important role is determining the entrapment of drug and morphology of the system. On increasing the temperature of medium the entrapment efficiency of the drug increases till to optimum temperature.

\section{Entrapment efficiency}

The amount of free drug was measured by UV spectrophotometer at $289 \mathrm{~nm}$. Optimized emulsion entrapment efficiency was $81.1 \%$

\section{Droplet size of Nanoemulsion}

Droplets of nanoemulsion was found around 380 nm shown in Figure 2.

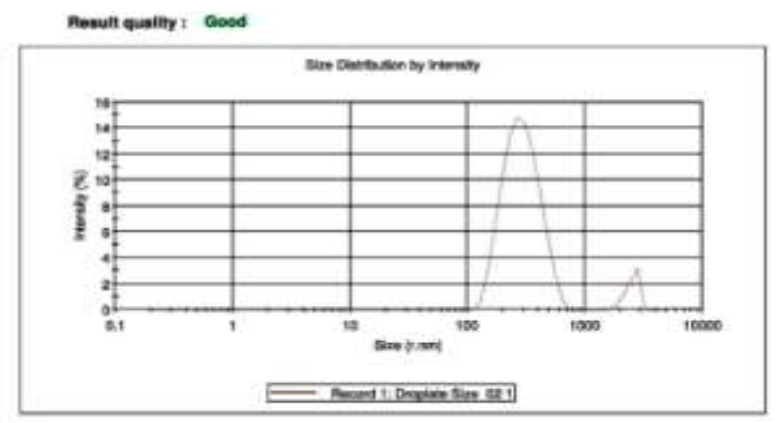

Fig. 2: Droplet size of optimized nanoemulsion

\section{Viscosity}

Viscosity of optimized formulation was $7 \mathrm{Cp}$ which is comfortable viscosity for ocular administration.

pH

$\mathrm{pH}$ of optimized formulation was 7.2-7.4 which is comfortable for ocular administration of dosage form.

\section{Scanning Electron microscopy}

SEM showed droplets size oval to spherical in shape with size of $300 \mathrm{~nm}$ which justify termn nano emulsion.

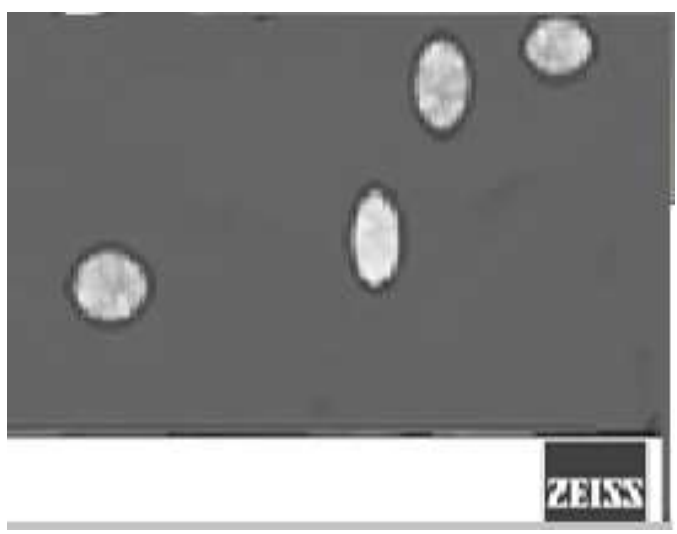

Fig. 3: SEM of optimized formulation 
Microscopic image taken by Phase contrast microscope and droplets are found oval to spherical in shape.

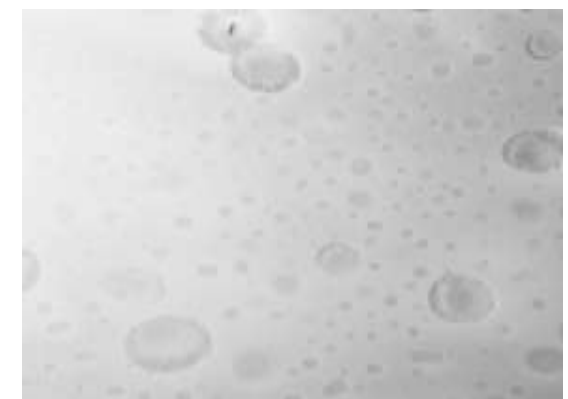

Fig. 4: Microsopy of formulation

\section{In vitro drug release}

For the estimation of the in-vitro drug release from the emulsion. Marketed moxifloxacin hydrochloride formulation was a used of as a model drug. The cumulative percentage release was $88 \%$ from the nanoemulsion over a period of $6 \mathrm{hr}$ shown in

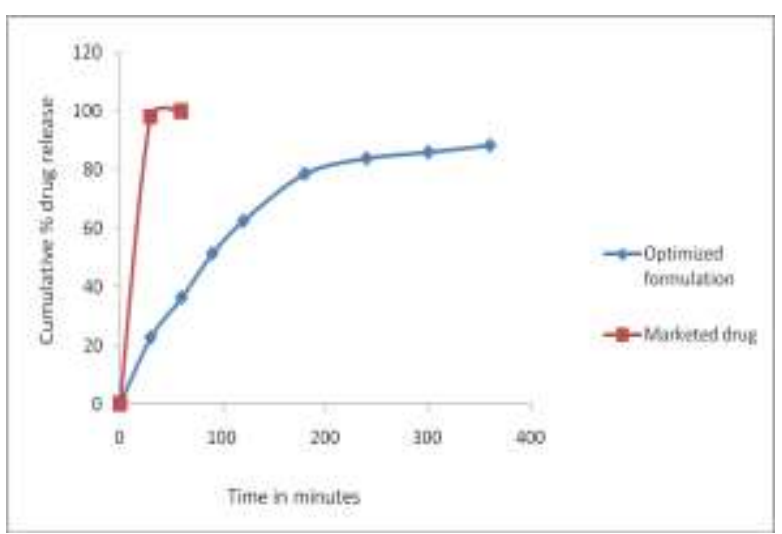

Fig.5: In vitro release of optimized and marketed formulation

The storage stability of formulation was checked in terms of change in viscosity, $\mathrm{pH}$, Creaming \& cracking Nanoemulsion sample at $4^{\circ} \mathrm{C} \& 25^{\circ} \mathrm{C}$ over a period of 2 months. The $\mathrm{pH}$ of optimized formulation was in range of 7.4-6.2. It has been observed that the no major changes reported by the storage temperature with time. The viscosity of emulsion sample stored at $4^{\circ} \mathrm{C} \& 25^{\circ} \mathrm{C}$ not influences more with time. Data shown in table 2 and 3. No significant creaming and cracking was observed, system gets re-dispersed after little shaking.

Table: 2 Influence of temperature $4^{\circ} \mathrm{C}$ on viscosity and $\mathrm{pH}$

\begin{tabular}{|l|l|l|}
\hline Time (days) & Viscosity $(\mathbf{c P})$ & $\mathbf{p H}$ \\
\hline 0 & $7.8 \pm 0.9$ & $7.1 \pm 0.1$ \\
\hline 15 & $7.2 \pm 0.7$ & $7.4 \pm 0.3$ \\
\hline 30 & $7.0 \pm 0.8$ & $7.2 \pm 0.4$ \\
\hline 60 & $7.3 \pm 0.9$ & $7.3 \pm 0.1$ \\
\hline
\end{tabular}

Table: 3 Influence of temperature $25{ }^{\circ} \mathrm{C}$ on viscosity and $\mathrm{pH}$

\begin{tabular}{|l|l|l|}
\hline $\begin{array}{l}\text { Time } \\
\text { (days) }\end{array}$ & Viscosity $(\mathbf{c P})$ & $\mathbf{p H}$ \\
\hline 0 & $7.1 \pm 0.8$ & $7.1 \pm 0.1$ \\
\hline 15 & $7.0 \pm 0.4$ & $7.3 \pm 0.1$ \\
\hline 30 & $6.0 \pm 0.2$ & $7.2 \pm 0.3$ \\
\hline 60 & $6.5 \pm 0.1$ & $6.2 \pm 0.2$ \\
\hline
\end{tabular}

\section{CONCLUSION}

Based on the results observed from SEM, Particle size, Viscosity $\mathrm{pH}$ and in vitro release study, designed formulation can be a good dosage form for topical delivery of drug for eyes. However efficacy of formulation depends on ocular experimentation on animal model.

\section{Acknowledgement}

Author is thankful to SIRT-Pharmacy and SIRTS Pharmacy for successful conduction of experimentation and data compilation of project.

Conflict of Interest: Declared none

\section{REFERENCES}

1. Pandey D, Singh R, Jain S and Jain D. Ofloxacin ion pairing within submicron emulsion: a potential approach for ocular delivery. Indian Drugs, 2019; 56(04):56-63.

2. Deshmukh RL, Multiple emulsion: Strategic and technology, Asian Journal of Pharmaceutical Education and Technology, 2014; 2(2): 1-19. 11. Pandey D, Kesharwani P, Jain D. Entrapment of drug-sorbate complex in submicron emulsion: A potential approach to improve antimicrobial activity in bacterial corneal infection. J Drug Deliv Sci Tec, 2019; 49:455-462.

3. Jain V, Gupta S, Garg D, Mishra P. Ciprofloxacin surf-plexes in sub-micron emulsions: A novel approach to improve payload efficiency and antimicrobial efficacy. Int J Pharm, 2011; 56-43.

4. Somchit M, Reezal I, Nur IE, Mutalib A. In vitro antimicrobial activity of ethanol and water extracts of Cassia alata. J Ethnopharmacol, 2003; 84(1):1-4.

5. Bhatia N, Pandit S, Agrawal S and Gupta D. A review on multiple emulsions. International Journal of Pharmaceutical Erudition 2013; 3(2): 22-30.

6. Ahuja A, Ali J, Baboota S, Faisal M, Shakeell F, Shafiq S, Stability evaluation of Celecoxib Nanoemulsion containing Tween 80, Thai J Pharm Sci, 2008; 32: 4-9. 
7. Kriwet K, Muller-Goymann, CC, Diclofenac release from phospholipid drug systems and permeation through excised human stratum corneum. International Journal of Pharmaceutics, 125: 231-42.

8. D. Pandey, D. Jain formulation and evaluation of submicron emulsion containing entrapped fluoroquinolone for ocular delivery. Asian Journal of Pharmaceutical and clinical research, 2018; 11(7): 431-35.

9. Levy MY, Benita S. Drug release from submicronized o/w emulsion: A new in vitro kinetic evaluation model, International Journal of Pharmaceutics, 1990; 66: 29-37.

10. Pandey $\mathrm{d}$ and jain $\mathrm{d}$ "improved ciprofloxacin penetration in goat eyes using complexation technique". Asian journal of pharmaceutical and clinical research, 2019;12(8):168-71. 